\title{
New Generation Artificial Intelligence-driven Intelligent Manufacturing (NGAIIM)
}

\author{
Bonu L1 \\ Beijing University of \\ Aeronautics and \\ Astronautics \\ Beijing, China \\ bohuli@buaa.edu.cn
}

\author{
Auaong unal \\ CASICloud Co. \\ Ltd. \\ Beijing, China, \\ xdchai@263.net \\ Baocun nou \\ Beijing Simulation \\ Center, \\ Beijing, China \\ houbc2015@sina.com
}

\author{
Lin Zhang \\ Beijing University of \\ Aeronautics and \\ Astronautics \\ Beijing, China \\ zhanglin@buaa.edu.cn
}

\author{
Jiehan Zhou \\ (Member IEEE) \\ University of Oulu, \\ Finland \\ Oulu, Finland \\ jiehan.zhou@oulu.fi
}

\author{
Yang Liu \\ Hangtian Zhizao Co. \\ Ltd. \\ Beijing, China \\ 289503799@qq.com
}

\begin{abstract}
In order to embrace new generation artificial intelligence to upgrade manufacturing industry, we propose the concept of new generation artificial intelligence-driven intelligent manufacturing (NGAIIM), which is a new manufacturing paradigm integrating human/machine/environment/information into product lifecycle activities. First, we introduce new generation artificial intelligence. Second, we present NGAIIM connotation, NGAIIM architecture and its technology system. Then, we examine a NGAIIM use case - CASICloud. Finally, we provide suggestions for directing the NGAIIM development.

Keywords: Artificial Intelligence, New Generation Artificial Intelligence, Intelligent manufacturing, Big Data
\end{abstract}

\section{INTRODUCTION}

The future of industry will reward innovation, openness, sharing, green manufacturing, and the development of human talent. To promote these long-term development goals, China proposed "Made in China 2025" for building a strong nation by 2025, whose overall strategy is innovation-driven, quality-first, green development, structural optimization, and talent-based for fully leveraging emerging technologies [1]. These technologies are highlighted by the Internet of Things (IoT) [2], big data [3], cloud computing [4], 3D printing [5], Blockchain [6], and new generation artificial intelligence (NGAI) [7], etc. With the rapid development of mobile terminals, sensor networks [8], service-oriented computing [9], vehicle network [10], traditional AI is being overtaken by new generation artificial intelligence, which takes on features of big data intelligence, group intelligence, hybrid human-computer intelligence, cross-media reasoning, etc.

As one of the mandated technologies driving "Made in China 2025", NGAI promotes the transformation of production-oriented manufacturing to service-oriented manufacturing through interconnecting the physical and information worlds. NGAI is expected to support deep intelligence insights into the entire product lifecycle, from demand acquisition, design, manufacturing and operating services, maintenance and recycling. In addition, NGAI plays an important role in network collaborative manufacturing, and personalized customization, etc. For example, NGAI enables intelligent control, monitoring, and failure prediction in a product machining process.

There are few reports discussing the relationship between new generation artificial intelligence and intelligent manufacturing. We propose the concept of NGAI-driven intelligent manufacturing (NGAIIM) for accelerating the utilization of NGAI into intelligent manufacturing. In addition, we introduce the progress of NGAIIM with the research and development of CASICloud [11].

The remainder of the paper is organized as follows. Section 2 introduces NGAI. Section 3 defines NGAIIM and identifies its features. Section 4 presents the NGAIIM system architecture. Section 5 presents technologies enabling NGAIIM. Section 6 introduces our NGAIIM practice on CAISCloud. Section 7 concludes the paper and gives suggestions directing the future NGAIIM development.

\section{NEW GENERATION ARTIFICIAL INTELLIGENCE[12]}

\section{A. Definition}

After experiencing its birth, development, and recent commercialization, artificial intelligence is booming and entering a new rapid development stage, which is called New Generation Artificial Intelligence (NGAI). In this paper, we define new generation artificial intelligence as the artificial intelligence based on the new information environment, new computing technologies, and new application development goals. The new information environment is comprised of the continually expanding Internet, increasing mobile devices, social media and networks, sensor networks, etc. New computing technologies include big data, high-performance computing, and new approaches and algorithms for machine learning, etc. New application development goals include smart cities, digital economy, intelligent manufacturing, smart healthcare, smart homes, intelligent vehicles and so on.

\section{B. Driving forces}

With the rapid evolution of information communication technology such as mobile Internet, big data, and cloud computing, NGAI has the following driving forces:

- Rapidly expanding big data. Thanks to the proliferation of the Internet and various cyber-physical systems, the amount of data is dramatically growing, which provides the opportunity for insights through deep learning.

- Rapidly increasing data processing speed and improving computing power. The emergence of specific chips for artificial intelligence such as graphics processing units (GPUs), Neuronal Processing Units (NPUs), and Field-programmable gate arrays (FPGAs) enables 
machines to process big data faster.

- Deep learning breakthroughs. Deep learning has achieved state-of-the-art results across a range of difficult domains over the past years [13-15]. Deep learning algorithms have been widely used in natural language processing [16], speech processing [17], and computer vision [18, 19]. Supervised learning is evolving into semi-supervised and unsupervised learning. Machines are now beginning to distinguish objects and translate speech in real time.

- Increasing capital investment. Increased capital investing artificial intelligence helps the AI industry and start-up companies to rapidly and continuously escalate the AI business.

\section{NGAI characteristics}

Driven by the above-mentioned forces, NGAI is evolving from simulating human intelligence to assisting human intelligence through the interconnecting of machines, people, and the Internet. NGAI has the following major characteristics:

Big data-fueled artificial intelligence. Increasingly, big data is the cornerstone for the renaissance of artificial intelligence. Unlike early reasoning-based artificial intelligence, NGAI is powered by big data for building optimal learning models through inputting big training data into the model and determining optimal parameters for the model. For example, after trained with thousands of human-played games and self-playing millions of games [20], AlphaGo defeated the top Go player in the world.

Cross-media artificial intelligence. NGAI is oriented to comprehensively process text, image, voice, and video information to gradually approximate human intelligence. NGAI aims to imitate human beings' perceptions such as vision, speaking, and listening and achieve the functions of recognition, reasoning, self-design, prediction, etc.

Web-based group artificial intelligence. Intelligence could be enhanced and enriched through grouping the participation and interaction of individuals on the Internet. NGAI is expected to create a multi-agent synergistic group intelligence through studying group intelligence theory, and organization, incentive mechanisms, etc.

Hybrid human-machine synergized artificial intelligence. NGAI takes account of the people in the loop of using artificial intelligence, and this takes advantage of machine intelligence in search, calculation, storage, optimization, and human intelligence in perception, reasoning, induction, and learning.

\section{NGAIIM DEFINITION AND IDENTIFICATION}

\section{A. NGAIIM definition}

NGAIIM aims to realize manufacturing intelligence in product design, process, manufacturing, quality control, production management and service with the use of data modeling, processing, analysis, visualization and management.
NGAIIM promises to achieve efficient, high quality, costsaving, green, and flexible production, thereby increasing the market competitiveness for enterprises. NGAIIM features autonomous and intelligent perception, interconnection, collaboration, machine learning, data analysis, decisionmaking, control and execution, all interacting with human, machine, environment, and the information involved in all product lifecycle activities.

\section{B. NGAIIM identification}

From a technical perspective, combining the Internet of Things, artificial intelligence, NGAIIM mainly examines and visualizes big data collected from a product lifecycle, and realizes intelligent information systems in all product phases, including enterprise resource planning (ERP), manufacturing execution system (MES), Supply Chain Management (SCM), Product Lifecycle Management (PLM), Customer Relationship Management (CRM), and Process Control System, etc. Based on the ubiquitous network, with the integration of manufacturing know-how and information communication technology (ICT), NGAIIM is expected to form a unified user-centered cloud platform that manages manufacturing resources, products, and capabilities, and enables users to efficiently access manufacturing resources ondemand and anywhere through the mobile or static terminals.

From an application perspective, NGAIIM supports applications such as visual description, control, and decisionmaking based on data processing and management so as to enable intelligent design, intelligent manufacturing, networked collaborative manufacturing, intelligent service, and personalized customization, etc. NGAIIM stores the production results in the form of standardized data, and continuously optimizes production lifecycle management and cooperation from production equipment to control level, from workshop management to enterprise operation level, all along the industrial business chain.

From a resource management perspective, NGAIIM aims to optimize production resource and their flows involving in product lifecycle activities. Resources consist of people, technology/equipment, management, data, materials, funds (so called "six elements"). Flow consists of human, technology, management, data, logistics, and capital flow (so called "six flows").

From the manufacturing perspective, NGAIIM creates a new intelligent manufacturing mode characterized by being user-centered, human/machine/environment/information fusion, interconnected, service-oriented, personalized customization, flexible, and socialized.

From the industry ecosystem perspective, NGAIIM-based ecosystem is ubiquitous interconnection, data-driven, shared services, cross-border integration, and innovation.

\section{NGAIIM ARCHITECTURE}

Fig. 1 illustrates NGAIIM system architecture, which consists of four layers. 


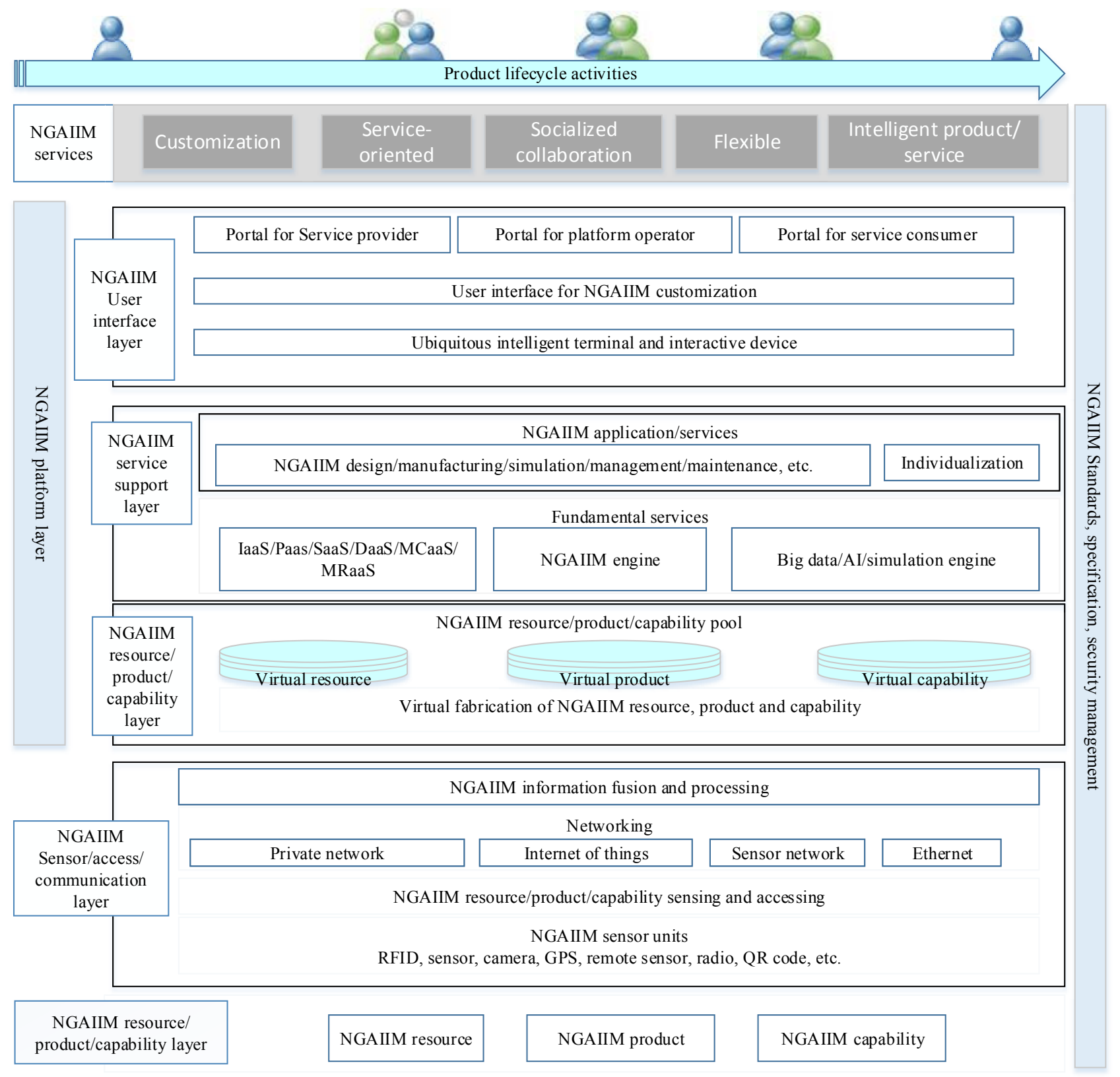

Figure. 1. NGAIIM system architecture

(1) Resources/capabilities/products layer. Includes various software/hardware resources, professional capabilities, and products.

(2) Sensing/access/communications layer. Connects various types of physical hardware/software resources/capabilities/products to networks through sensing, IoT, and terminal technology. The ubiquitous awareness and interconnection provide support for capability virtualization and service invocation. At the same time, this layer provides support for edge computing to process data locally.

(3) Platform layer. Consists of three sub-layers: virtualization, service support, and user interface. The user interface layer provides portals for the service provider, platform operator, and service consumer. The service support layer provides fundamental services such as IaaS and simulation engine for application development. The Virtualization layer virtualizes resources/product/capability into service pool for responding to intelligent manufacturing activity requests.

(4) Service layer. Provides a new mode of intelligent manufacturing which is characterized by being highly interconnected and collaborative, service-oriented, flexible, social, intelligent, and personally customizable.

\section{NGAIIM TECHNOLOGY SYSTEM}

Fig. 2 illustrates the NGAIIM technology system. It mainly consists of 


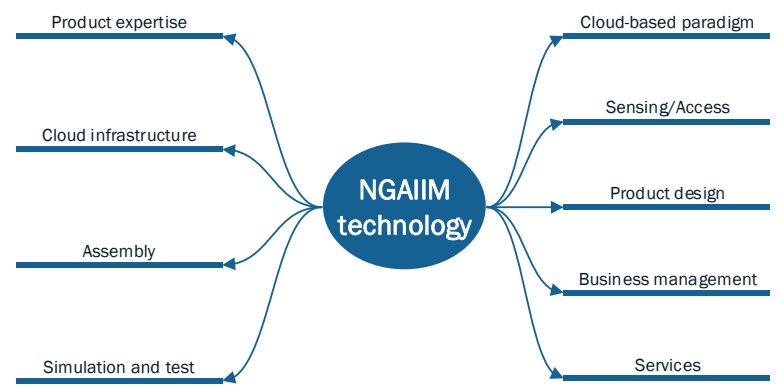

Figure 2. NGAIIM technology system

- Cloud-based paradigm (e.g., NGAIIM-driven business model, system integration, system architecture, standardization, system development and application, and security),

- Sensing/access technology (e.g. Internet, IoT, vehicle network, etc.),

- Product design (e.g., crowd intelligence-oriented design, cross media-design, etc.),

- Business management (e.g., big data-driven project management, enterprise management, quality management, etc.)

- Service technology (e.g., pre-sale/post-sale maintenance, value-added services, etc.)

- $\quad$ Product expertise (e.g., service-oriented)

- Cloud infrastructure (e.g., virtualization, simulation engine, pervasive human computer interface, etc.)

- Assembly (e.g., industrial robotics, 3D printing, etc.)
- $\quad$ Simulation and testing (e.g. big data-driven modeling and simulation, cloud-based simulation, etc.).

\section{NGAIIM USE CASE - CASICLOUD}

Based on our R\&D on the Chinese aerospace industry and the pioneering experience in the industrial Internet, we applied NGAIIM into the development of the first China's industrial Internet platform: CASICloud [21]. This platform efficiently integrates and shares high, medium, and low-end industrial resources at home and abroad. CASICloud provides core technologies of resource virtualization, capability-based service, and product-based service for intelligent manufacturing.

China's manufacturing currently coexists at different levels of development: Industry 1.0, Industry 2.0, and Industry 3.0 enterprises. CASICloud can be used by all industry levels to provide enterprises, governments, and entrepreneurs with a comprehensive "Internet + Intelligent Manufacturing" solution covering the entire industry chain and production life. NGAIIM is expected to leverage Chinese industry 1.0/2.0/3.0/4.0. This section focuses on the architecture of CASICloud, its artificial intelligence system, and the user cases.

\section{A. CASICloud system architecture}

Fig. 3 illustrates CASICloud architecture, which consists of Device, IoT, Access, INDICS platform [22, 23], Service and application layers. The INDICS platform provides small to medium enterprises (SME) with successful access to intelligent factories, the industrial cloud, industrial big data, and intelligent services.

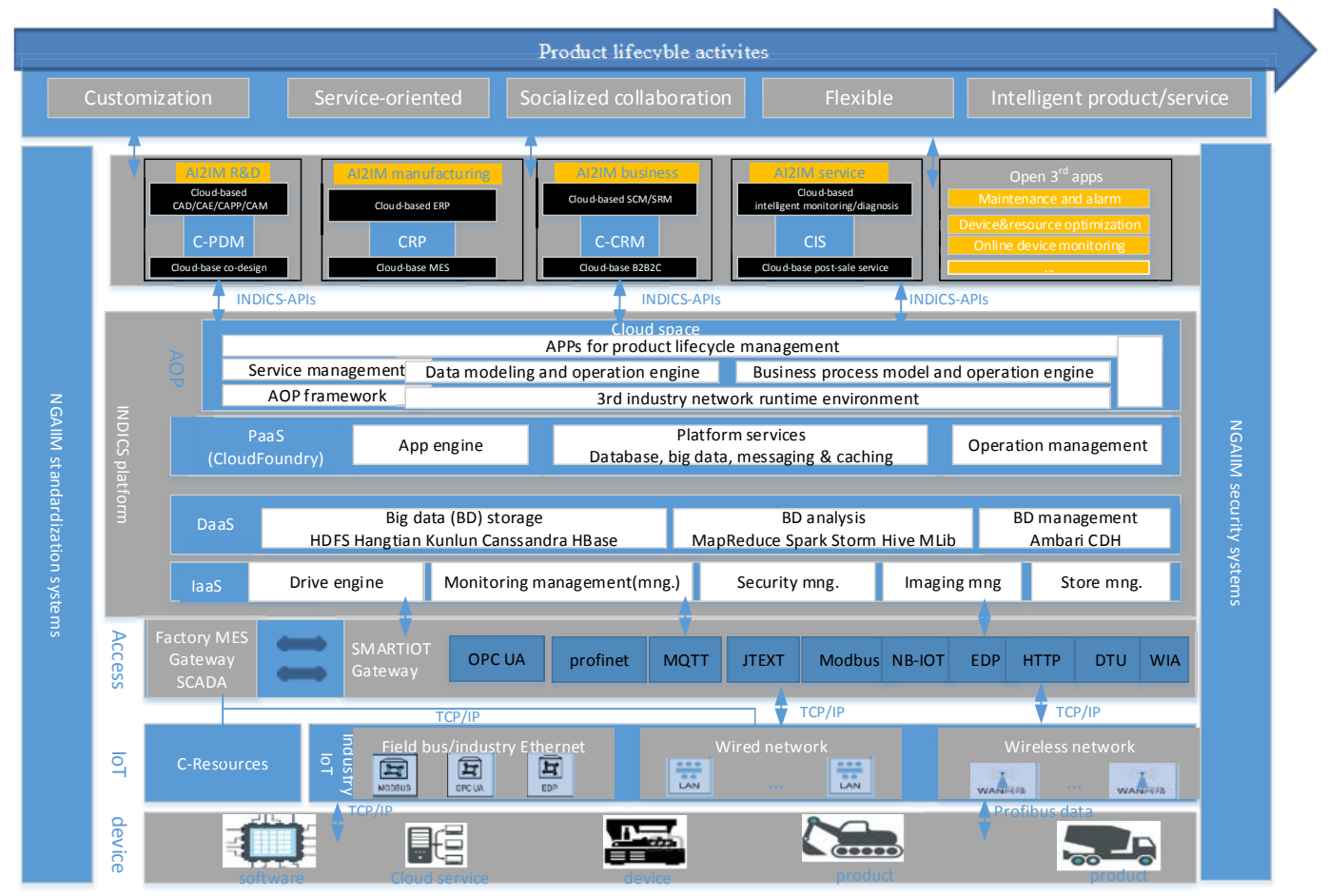

Figure. 3. CASICloud architecture 


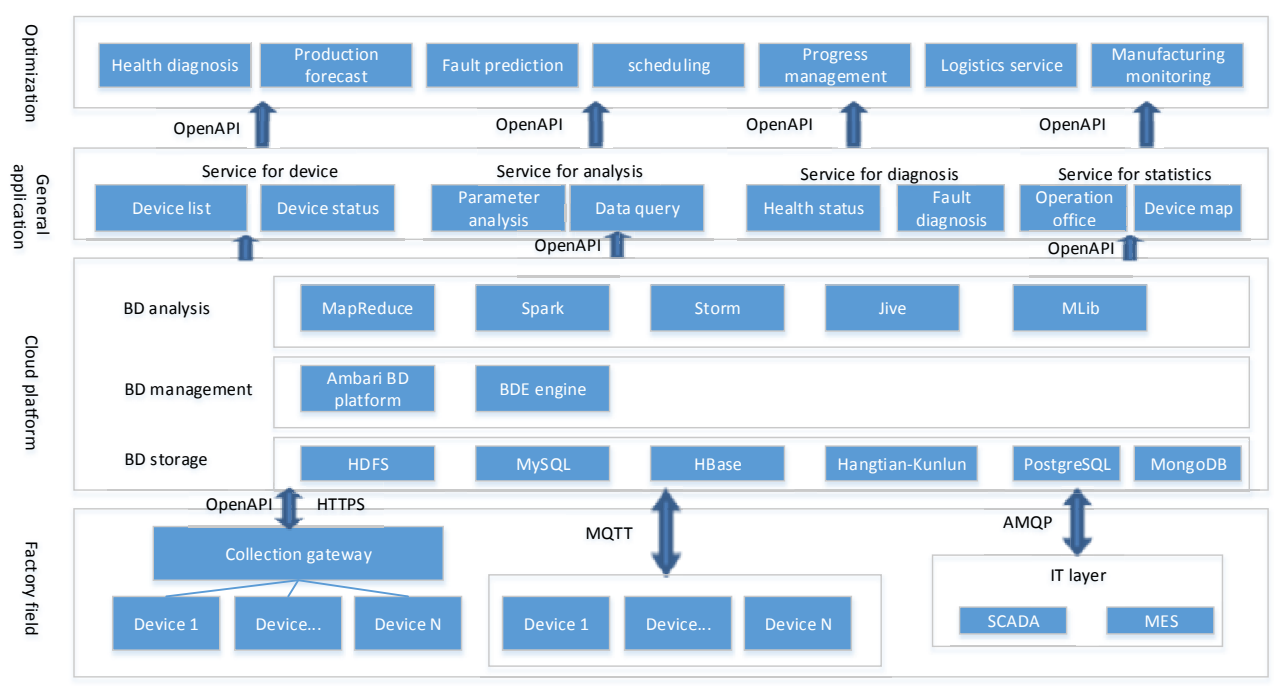

Figure 4. CASICloud big data/AI system

\section{B. user cases}

1) CASICloud big data/AI system

Figure 4 presents CASICloud big data/AI system, an industrial big data/artificial intelligence subsystem, which consists of optimization, general application, cloud-based big data platform and factory field layers.

2) Key technology for CASICloud big data

The key technology covers big data integration, cleaning, storage and management, analysis and mining, visualization, standards and quality, and big data security [24].

In the big data integration and cleaning, we used ExtractTransform-Load (ETL) to clean and convert the redundant and dirty data created by operating equipment so as to achieve effective access to equipment data. We monitored data quality for massive enterprise's data storage and management based on time series and columnar database, distributed historical data storage, and document database.

We implemented off-line analysis and mining for overall equipment effectiveness (OEE) based on Hadoop. We implemented real-time fault diagnosis based on Storm. We realized the visualization of enterprise data based on Business Intelligence (BI). We studied data encryption, backup, and access authority to maximize data security.

With the above big data intelligence technology, CASICloud is able to upgrade and enhance the competitiveness of enterprises through informatization, industrial IoT device interconnection, cross-border data exchange, business optimization, etc.

\section{3) Applications}

Currently, the industrial big data subsystem of the CASICloud has achieved impressive results in $R \& D$, production, operation and maintenance. For example, during aerospace cable design, we saved the development time using the cable design tool E3 based on the historical big data and integrated flight performance data.

Another example is Hangtian-based production scheduling, which collects and monitors multiple $\mathrm{CNC}$ machining centers through cloud-based Manufacturing Execution System (MES), and schedules and optimizes production resources across different locations for large-scale cooperation of aerospace products.

\section{CONCLUSION AND FUTURE}

Big data and artificial intelligence are key technologies for intelligent manufacturing. Deep learning plays an indispensable role in intelligent design, intelligent production, networked manufacturing, intelligent services, and personalized customization. In this paper, we introduce the new generation artificial intelligence that brings the concept of NGAIIM; we examine NGAIIM from the perspectives of technology, application, resource management and industry ecosystem; we present the NGAIIM architecture and outline its technology system; in addition, we present a user case of NGAIIM-driven CASICloud.

NGAI in intelligent manufacturing is still in its infancy. We take the lead in studying NGAIIM to integrate NGAI into R\&D on manufacturing intelligence. At present, NGAIIM faces many issues, such as the standardization of manufacturing product big data, IoT equipment control technology, seamless information integration, and big data security. In the future, we will further research and develop NGAIIM by applying the latest technologies such as cloud computing, the Internet of Things, artificial intelligence, and $5 \mathrm{G}$ to the following three principles:

(1) NGAIIM is a new intelligent manufacturing mode, which fully leverages the upgraded technology of Internet, big data, artificial intelligence. NGAIIM presents a new technology that accelerates the development of intelligent manufacturing.

(2) NGAIIM is under development. Its development needs the coordination and collaboration of NGAIIM technology, NGAIIM application, and NGAIIM industry. Its development path should persist in the innovation-driven integration of industrialization and informatization. 
(3) NGAIIM development and implementation also require cooperation and exchanges between at home and abroad. At the same time, NGAIIM should pay attention to the differences of various industries and enterprises.

\section{REFERENCES}

[1] Made in China 2025: global ambitions built on local protections https://www.uschamber.com/sites/default/files/final_made_in_china_2 025_report_full.pdf. Access by 17.3. 2018

[2] J. Zhou, T. Leppänen, E. Harjula,M. Ylianttila, T. Ojala, C, Yu, H. Jin. "CloudThings: A common architecture for integrating the Internet of Things with Cloud Computing" Proceedings of the 2013 IEEE 17th International Conference on Computer Supported Cooperative Work in Design (CSCWD), Whistler, BC, 2013, pp. 651-657.

[3] W. Zhang, Y. Zhang, J. Zhai, D. Zhao, L. Xu, J. Zhou, Z. Li, S. Yang, Multi-source data fusion using deep learning for smart refrigerators, Computers in Industry, Volume 95,2018,Pages 15-21,

[4] W. Zhang, L. Chen, X. Liu, Q. Lu, P. Zhang, \& Yang, S. An OSGibased flexible and adaptive pervasive cloud infrastructure. Science China Information Sciences, 57(3), 1-11, 2014.

[5] B.Mohajeri, T.Nyberg, J.Karjalainen, T.Tukiainen, M.Nelson, X.Shang G.Xiong. The impact of social manufacturing on the value chain model in the apparel industry. In proceedings of IEEE Service Operations and Logistics, and Informatics (SOLI), pp. 378-381, 2014.

[6] Y.Yuan, F. Y. Wang. Towards blockchain-based intelligent transportation systems. In proceedings of IEEE Intelligent Transportation Systems (ITSC), pp. 2663-2668, 2016.

[7] Y. Pan. Heading toward artificial intelligence 2.0. Engineering, 2(4), 409-413. 2016.

[8] J. Z. Sun, J. Zhou. Extending SQL to support QoS and semantic data gathering in WSN. In proceeding of International Conference on Software Engineering and Data Mining, Chengdu, China, pp. 86-90, 2010.

[9] J. Zhou, J. Riekki. Context-aware pervasive service composition. In proceeding of Intelligent Systems, Modelling and Simulation (ISMS), pp. 437-442, 2010.

[10] L. Li, F. Y. Wang, Q. Zhou. Integrated longitudinal and lateral tire/road friction modeling and monitoring for vehicle motion control. IEEE Transactions on intelligent transportation systems, 7(1), 1-19. 2006.

[11] CASICloud. Homepage. http://www.casicloud.com/, accessed by 18.03.2018.

[12] Y. PAN. Special issue on artificial intelligence 2.0: theories and applications. Frontier of Information Technology and Electronic Engineering. 2018 19(1):1-2

[13] H. Robert. Deep Learning. MIT Technology Review. https://www.technologyreview.com/s/513696/deep-learning/, accessed by 18.03 .2018 .

[14] W. Zhang, Y. Zhang, J. Zhai, D. Zhao, L. Xu, J. Zhou, Z. Li. Multisource data fusion using deep learning for smart refrigerators. Computers in Industry, vol. 95, pp.15-21,2018.

[15] W. Zhang, W. Guo, X. Liu, Y. Liu, J. Zhou, B. Li, Q. Lu. LSTM-Based Analysis of Industrial IoT Equipment. IEEE Access, vol. 6, pp. 23551 23560.2018

[16] Y. LeCun, Y. Bengio, \& G. Hinton. Deep learning. Nature, 521(7553), 436, 2015.

[17] L. Zhu, L. Chen, D. Zhao, J. Zhou, W. Zhang. Emotion recognition from Chinese speech for smart affective services using a combination of SVM and DBN. Sensors, 17(7), 1694, 2017.

[18] W. Zhang, D. Zhao, L. Xu, Z. Li, W. Gong, J. Zhou. Distributed embedded deep learning based real-time video processing. In IEEE International Conference on Systems, Man, and Cybernetics (SMC), Budapest, pp. 001945-001950, 2016.

[19] W. Zhang, Z. Wang, X. Liu, H. Sun, J. Zhou, Y. Liu, W Gong. Deep learning-based real-time fine-grained pedestrian recognition using stream processing. IET Intelligent Transport Systems, 2018

[20] E. Gibney. Google AI algorithm masters ancient game of Go. Nature News, 529(7587), 445, 2016.

[21] Y. Wei. X. Chai. Industrial practice of Internet technology [M]. Electronic Industry Press, 2017.

[22] CASICloud. INDICS. http://intl.indics.com/, accessed by 18.03.2018
[23] X. Chai, B. Hou, P. Zou, J. Zeng, J. Zhou. INDICS: An Industrial Internet Platform. In IEEE International Conference on Cloud and Big Data Computing, Guangzhou, China, Octobor 8-12, 2018.

[24] B. Hou, X. Chai. Smart Manufacturing Cloud - A Kind of Smart Manufacturing System based on Industrial Big Data and Artificial Intelligence. Beijing: The Future Industrial Intelligent Summit, 2017. 